\title{
Thermodynamic Equilibrium Conditions of Graphene Films on SiC
}

\author{
Lydia Nemec, Volker Blum, Patrick Rinke, and Matthias Scheffler \\ Fritz-Haber-Institut der Max-Planck-Gesellschaft, Faradayweg 4-6, D-14195 Berlin, Germany
}

(Received 29 April 2013; published 6 August 2013)

\begin{abstract}
First-principles surface phase diagrams reveal that epitaxial monolayer graphene films on the Si side of $3 \mathrm{C}-\mathrm{SiC}(111)$ can exist as thermodynamically stable phases in a narrow range of experimentally controllable conditions, defining a path to the highest quality graphene films. Our calculations are based on a van der Waals corrected density functional. The full, experimentally observed $(6 \sqrt{3} \times 6 \sqrt{3})-R 30^{\circ}$ supercells for zero- to trilayer graphene are essential to describe the correct interface geometries and the relative stability of surface phases and possible defects.
\end{abstract}

DOI: 10.1103/PhysRevLett.111.065502

The growth of wafer-size graphene films on a semiconducting substrate is a first step towards graphene-based electronics. On the semiconductor $\mathrm{SiC}$, well-ordered graphene films can be grown directly by a simple process ( $\mathrm{Si}$ sublimation from the surface; see, e.g., Refs. [1-7]) and the standard tools of semiconductor technology can be used for further manipulation. Indeed, graphene-based devices and even integrated circuits $[8,9]$ have already been created on the $\mathrm{Si}$ side of SiC. However, controlling the precise thickness of graphene films is important to minimize the coexistence of monolayer graphene (MLG) and bilayer graphene (BLG) $[3,5,10,11]$. MLG areas exhibit no band gap, while BLG areas do [4]. A recent, joint experimental-theoretical study finds particularly high local resistances across monolayerbilayer graphene junctions [12], a possible contributing factor to low carrier mobilities in graphene on $\operatorname{SiC}(0001)[3,12]$.

Growing graphene on $\mathrm{SiC}$ is special in the sense that, instead of offering $\mathrm{C}$ from the gas phase, graphene areas form by controlled Si sublimation from the surface [1,2]. Graphene films grown under ultrahigh vacuum conditions are typically inhomogeneous [3,5]. To improve their quality is therefore a major and ongoing experimental goal $[3,5]$. In the past, the appearance of different phases was often interpreted $[5,13,14]$ as intuitive, successive intermediates formed by an outgoing Si flux, ultimately leading to bulklike graphite layers. If graphene films of various thicknesses were a result of a purely kinetically limited $\mathrm{Si}$ sublimation process (controlled by growth temperature and time), improving upon the homogeneity of the layer thickness would be difficult. It would be helpful if there were a set of thermodynamic equilibrium conditions (e.g., temperature $T$ and partial pressures $p$ of $\mathrm{Si}$ and $\mathrm{C}[15])$ at which a desired film thickness was thermodynamically stable over all others. As long as $T$ is sufficiently high to

Published by the American Physical Society under the terms of the Creative Commons Attribution 3.0 License. Further distribution of this work must maintain attribution to the author(s) and the published article's title, journal citation, and DOI.
PACS numbers: 61.48.Gh, 68.35.B-, 68.35.Md, 68.65.Pq

overcome the relevant kinetic barriers, large-scale ordered films of that thickness could then be formed simply by finding and maintaining $(p, T)$ near these equilibrium conditions.

Indeed, experimental evidence is mounting that controlled $(p, T)$ conditions do aid the growth of graphene on $\mathrm{SiC}$. Reference [16] shows that the carbon-rich "zero-layer graphene" (ZLG) or "buffer layer" precursor phase $[1,2,13,17]$ (not yet graphene) on the Si face is a reversible thermodynamic equilibrium phase at high $T$ and controlled disilane background pressure. Reversibility is much harder to demonstrate once a complete graphene plane is formed [18], but an increased growth temperature in an Ar buffer gas leads to much improved MLG film homogeneity [3]. Wafer-size MLG films are also reported for growth in a confined cavity that may retain a finite $\mathrm{Si}$ background pressure as Si evaporates from the surface [5]. Finally, a well-defined graphene precursor phase on the $\mathrm{C}$ face at finite disilane background pressure was reported recently [6]. What is still not clear, however, is whether MLG itself is an equilibrium phase under certain conditions. If so, one could ideally facilitate the growth of MLG but not BLG on $\mathrm{SiC}(111)$.

We here present first-principles theoretical evidence that such equilibrium conditions indeed exist for MLG (and, possibly, even BLG) on the Si face of $\mathrm{SiC}(111)$. We employ density-functional theory (DFT) using the van der Waals (vdW) corrected [19] Perdew-Burke-Ernzerhof (PBE) functional [20] (PBE + vdW). Strategies to include vdW effects are an active field (see, e.g., Refs. [21-26] and references therein). However, for the complex carbon-rich interfaces of interest here, the present incorporation of $\mathrm{vdW}$ terms [19] reflects the state of the art and no unambiguously quantitatively improved approach exists to our knowledge. For the relevant bulk phases, the impact of different standard functionals is well understood and systematic. Predicted lattice parameters (see also Supplemental Material [27]) for $3 \mathrm{C}-\mathrm{SiC}$ are within $1 \%$ of experiment: $a_{3 \mathrm{C}-\mathrm{SiC}}=4.33 \AA$ [local density approximation (LDA)], $4.38 \AA$ (PBE without vdW correction), $4.36 \AA$ (PBE + vdW), and $4.36 \AA$ (experiment [28]). Similarly small 
discrepancies arise for diamond $\mathrm{C}$ and for the in-plane lattice parameter of graphite: $a_{\text {graphite }}=2.45 \AA$ (LDA), $2.47 \AA$ (PBE), and $2.46 \AA$ (PBE + vdW). Zero-point corrections (ZPC) to the lattice parameters are also below $1 \%$ (see the Supplemental Material [27]). For the interplanar lattice parameter $c$ of graphite, $\mathrm{vdW}$ effects must be included into the PBE functional (6.65 $\mathrm{A}$ for LDA, $6.66 \AA$ for PBE $+\mathrm{vdW}$, but $8.65 \AA$ for PBE). All our calculations use the FHI-aims all-electron code [29,30] with "tight" numerical settings and the massively parallel ELPA eigensolver library [31] to guarantee accurate total energies for the very large structure sizes involved (see the Supplemental Material for details [27]).

In experiment, one encounters a series of phases on the $\mathrm{Si}$ side of 3C-SiC(111) [experimentally also observed on $4 \mathrm{H}-\mathrm{SiC}(0001)$ and $6 \mathrm{H}-\mathrm{SiC}(0001)]$ when going from Si-rich to C-rich conditions. In UHV, a $(3 \times 3)$ Si-rich layer [32] can be prepared. Upon Si sublimation, a simpler $(\sqrt{3} \times \sqrt{3})-R 30^{\circ}$ bulk-terminated surface with one adsorbed Si adatom per three unit cells follows [33,34]. Removing yet more $\mathrm{Si}$ creates a partially covalently bonded carbon layer, the ZLG phase, with a large, commensurate unit cell: one $(13 \times 13)$ honeycomb graphenelike supercell on a $(6 \sqrt{3} \times 6 \sqrt{3})-R 30^{\circ}$ mesh of the $\mathrm{SiC}$ substrate [1]. Compared to a graphene plane in graphite, the lattice match is almost strain free [experiment: $0.2 \%$ at $T=0 \mathrm{~K} \quad[28,35], \quad \mathrm{PBE}+\mathrm{vdW}: 0.1 \%$ (see the Supplemental Material [27])]. The ZLG phase does not yet exhibit the electronic properties of actual freestanding graphene [13]. Further heating detaches the ZLG C plane from the substrate to form MLG and a new C-rich layer underneath [36,37]. This process can be continued to successively form BLG [38] and multilayer graphene films. Importantly, our calculations address the graphenelike films in their experimentally observed, large commensurate $(6 \sqrt{3} \times 6 \sqrt{3})-R 30^{\circ}$ supercells, using slabs containing six Si-C bilayers under each reconstructed phase [1742 up to 2756 atoms for ZLG up to three-layer graphene (3LG)]. The top three SiC bilayers and all planes above are fully relaxed (residual energy gradients $8 \times 10^{-3} \mathrm{eV} / \AA$ or less).

Figures 1(a)-1(c) show ZLG, MLG, and BLG together with key geometry parameters predicted at the PBE + vdW level. Since all planes are corrugated [39-41], histograms for the atomic $z$ coordinates are included. The interface geometry stays essentially the same as more graphene planes are added. For MLG, we see a significant buckling of the topmost graphene layer ( $\approx 0.41 \AA$ between highest and lowest atoms). This buckling is qualitatively consistent with existing STM images $[11,42,43]$. In BLG, the corrugation is slightly reduced. The two top planes are buckled by $0.24 \AA$ and $0.32 \AA$, respectively. This buckling reflects some coupling to the covalently bonded $\mathrm{C}$-rich interface plane, which is much more corrugated $(\approx 0.8 \AA$ in our work, similar to experimental estimates $[44,45])$. The observed graphene interplanar distances near the interface

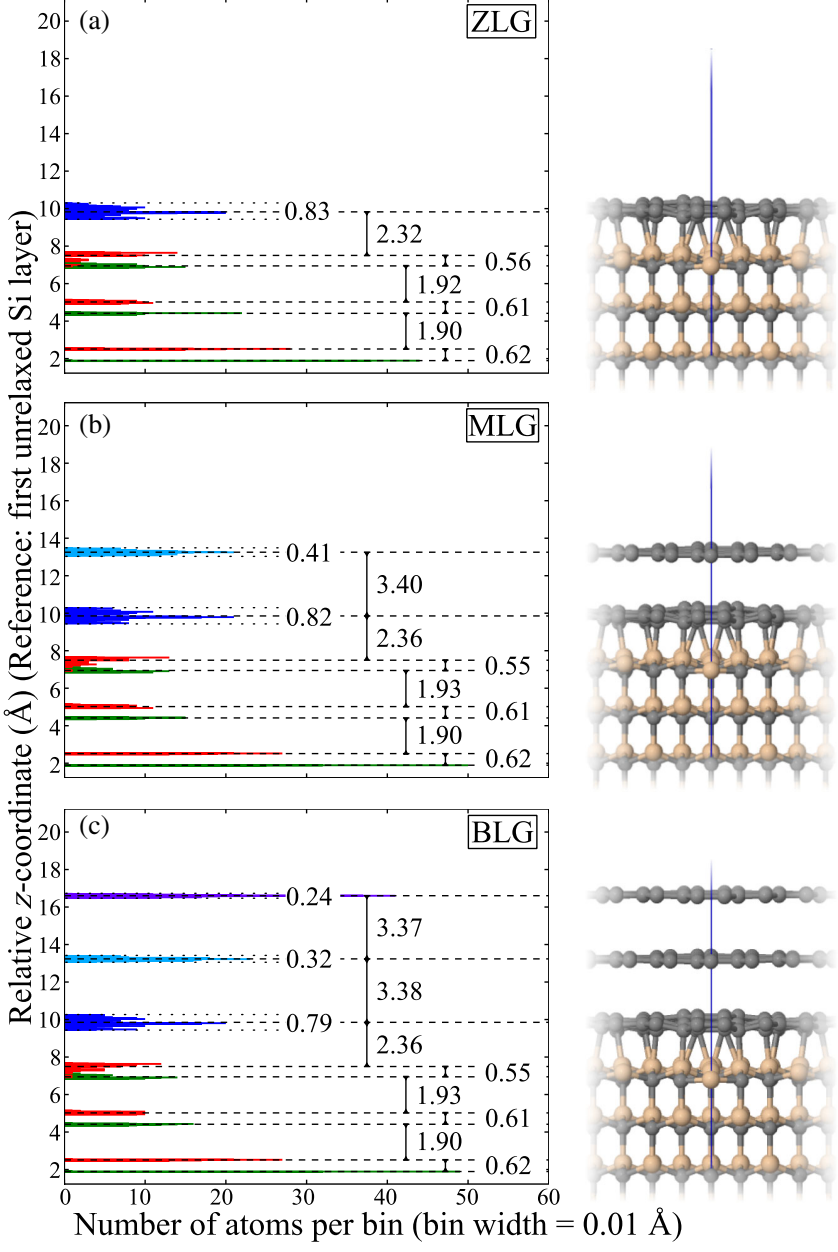

FIG. 1 (color online). (a)-(c): PBE + vdW calculated geometry of ZLG, MLG, and BLG on Si face 3C-SiC(111) and histograms of atomic $z$ coordinates (bin width: $0.01 \AA$ ) relative to the fourth $\mathrm{SiC}$ bilayer.

are slightly expanded compared to experimental bulk graphite (3.34 $\AA$ [35]) and in good qualitative agreement with estimates from scanning tunneling microscopy [43] and transmission electron microscopy [46].

We have also compared our findings to geometries for the straight PBE functional (no vdW correction), and for the LDA. In PBE, the $\mathrm{C}-\mathrm{C}$ interplanar distances are unphysically expanded (4.42 $\AA$ for MLG). In contrast, the LDA geometry of the carbon planes agrees qualitatively with $\mathrm{PBE}+\mathrm{vdW}$, although LDA incorporates no long-range vdW interactions. The first qualitative geometry difference between PBE + vdW and LDA appears in the Si part of the top SiC bilayer, where some $\mathrm{Si}$ atoms (those with dangling bonds pushing against the $\pi$-bonded parts of the C interface plane [47]) are located much deeper (by $\approx 0.3 \AA$ ) in PBE and PBE $+v d W$ than in LDA. A direct crystallographic verification would be desirable.

In a grand canonical formalism, equilibrium conditions for different surface phases can be represented by the 
chemical potentials of $\mathrm{C}$ and $\mathrm{Si}, \mu_{\mathrm{C}}$ and $\mu_{\mathrm{Si}}$. In experiment, $\mu_{\mathrm{C}}$ and $\mu_{\mathrm{Si}}$ can be manipulated, e.g., through the substrate temperature and background pressure $[5,6,15,16]$ of gases that supply $\mathrm{Si}$ or $\mathrm{C}$ [48]. Consider the surface energies $\gamma$ of a two-dimensional periodic $\mathrm{SiC}$ slab with a $\mathrm{C}$ face and a Si face. In the limit of sufficiently thick slabs, we have

$$
\gamma_{\mathrm{Si}-\text { face }}+\gamma_{\mathrm{C}-\text { face }}=\frac{1}{A}\left(E^{\text {slab }}-N_{\mathrm{Si}} \mu_{\mathrm{Si}}-N_{\mathrm{C}} \mu_{\mathrm{C}}\right) .
$$

$N_{\mathrm{Si}}$ and $N_{\mathrm{C}}$ denote the number of $\mathrm{Si}$ and $\mathrm{C}$ atoms in the slab, respectively. $A$ is the chosen area. We report surface energies in electron volts per $(1 \times 1) \mathrm{SiC}$ unit cell area. The letter $E$ denotes total energies for a given atomic geometry throughout this work. In all calculations, we choose a fixed $\mathrm{H}$-terminated $\mathrm{C}$-face geometry, which cancels out for all Si-face related surface energy differences.

It is important to note that Eq. (1) defines thermodynamic equilibrium conditions, but no conditions regarding growth kinetics. Thus, potential kinetic barriers do not enter the definition of thermodynamic stability according to the lowest surface energy given by Eq. (1). Of course, the actual surface morphology that forms in practice (finite growth time) still depends on temperature and the nature of kinetic barriers (see, e.g., Ref. [14] and references therein). For instance, higher growth temperatures that facilitate lateral surface diffusion processes are cited as the reason for improved graphene film quality in Refs. $[3,16]$. Still, Eq. (1) matters, since it will be much more difficult to drive a surface to a desired nonequilibrium phase than to an actual equilibrium phase.

The major experimental $(T, p)$ dependence during growth arises through the reservoirs of $\mathrm{Si}$ and $\mathrm{C}$, which define $\mu_{\mathrm{Si}}$ and $\mu_{\mathrm{C}}[15,16]$ (a small possible contribution of finite $T$ stresses at the growth conditions [49] must be kept in mind) [50]. Thus, a precise control of background gases as reservoirs (e.g., disilane [16]) is desirable, even if calibration variations [51] may require specific $(T, p)$ ranges to be adjusted for a given growth chamber.
In equilibrium with a stable $\mathrm{SiC}$ bulk, $\mu_{\mathrm{C}}$ and $\mu_{\mathrm{Si}}$ are linked through

$\mu_{\mathrm{Si}}+\mu_{\mathrm{C}}=2 E_{\mathrm{SiC}}^{\text {bulk }}=E_{\mathrm{C}}^{\text {bulk }}+E_{\mathrm{Si}}^{\text {bulk }}+2 \Delta H_{f}(\mathrm{SiC})$.

The energies are per atom. $\Delta H_{f}(\mathrm{SiC})$ is the formation enthalpy of $\mathrm{SiC}$ with respect to elemental $\mathrm{C}$ and $\mathrm{Si}$. The bulk phases define the chemical potential limits within which the $\mathrm{SiC}$ crystal is stable against decomposition into bulk Si or C: $\mu_{\mathrm{C}} \leq E_{\mathrm{C}}^{\text {bulk }}$ and $\mu_{\mathrm{Si}} \leq E_{\mathrm{Si}}^{\text {bulk }}$, leading to

$$
E_{\mathrm{C}}^{\text {bulk }}+\Delta H_{f}(\mathrm{SiC}) \leq \mu_{\mathrm{C}} \leq E_{\mathrm{C}}^{\text {bulk }}
$$

and analogous for $\mathrm{Si}$. The diamond structure for $\mathrm{Si}$ is the appropriate bulk phase. For $\mathrm{C}$, there is a close competition between diamond and graphite [52,53]. We thus include both phases in our analysis.

The experimentally reported energy difference between diamond and graphite at $T=0 \mathrm{~K}$ is $25 \mathrm{meV} /$ atom [52]. Based on the potential energy minima (no ZPC), graphite is more stable than diamond in $\mathrm{PBE}+\mathrm{vdW}$ by $60 \mathrm{meV} /$ atom [see Fig. 2(a)]. This is qualitatively consistent with the extrapolated experimental phase hierarchy. In plain PBE graphite is overstabilized by $130 \mathrm{meV} /$ atom. In LDA, both phases are similarly stable: considering only the potential energy surface, diamond is slightly more stable (by $12 \mathrm{meV}$ ), but already the inclusion of ZPC [53] would neutralize this balance (graphite more stable by $3 \mathrm{meV} /$ atom [54]).

Figure 2(a) shows the surface energies of the known surface phases of $\mathrm{SiC}$ (Si face) as a function of $\Delta \mu_{\mathrm{C}}=$ $\mu_{\mathrm{C}}-E_{\mathrm{C}}^{\text {bulk }}$ for PBE $+\mathrm{vdW}$. The most stable phase for a given value of $\Delta \mu_{\mathrm{C}}$ is that with the lowest surface energy. Going through Fig. 2(a) from left to right, we find the expected broad stability ranges for the Si-rich $(3 \times 3)$ and $(\sqrt{3} \times \sqrt{3})-R 30^{\circ}$ phases. Just before the C-rich limit (bulk graphite), there is a crossover first to ZLG, then to MLG, and even to a very narrow slice of the BLG phase. Finally, ABA-stacked 3LG crosses BLG within $1 \mathrm{meV}$ of bulk graphite.
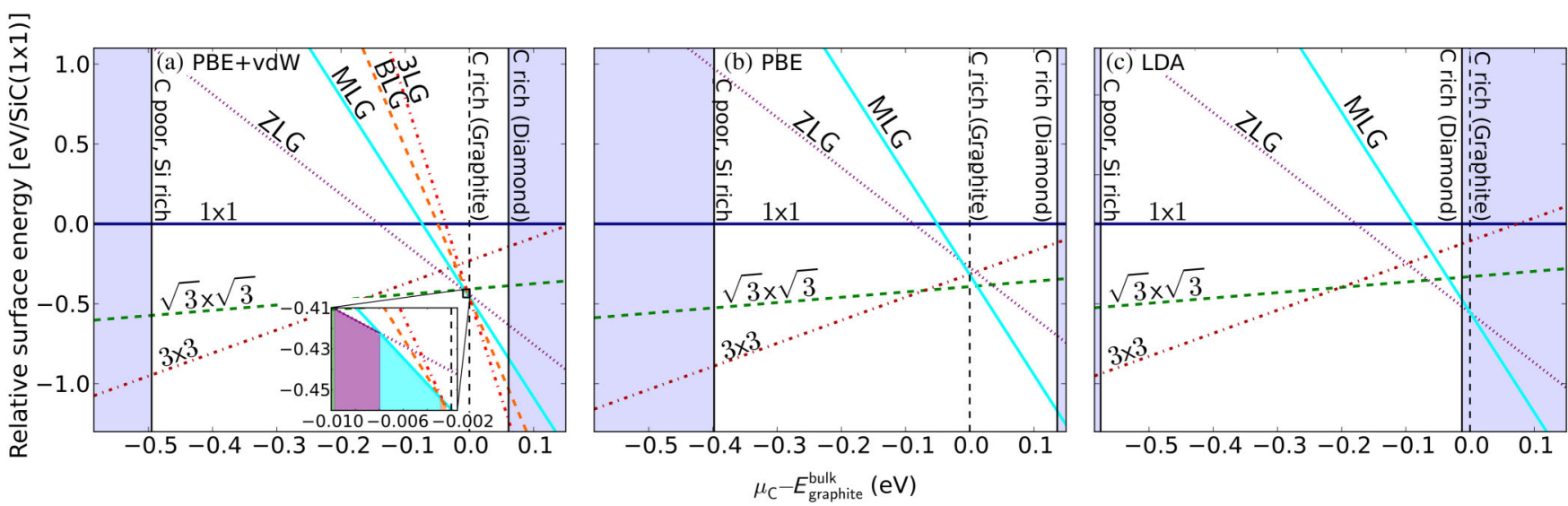

FIG. 2 (color online). Surface energies of different phases of Si-face $3 \mathrm{C}-\mathrm{SiC}(111)$ relative to $(1 \times 1)$, vs $\mu_{\mathrm{C}}$. Areas outside the limits set by Eq. (3) are shaded. (a) PBE + vdW, (b) PBE, (c) LDA. 
While the stability ranges are narrow (see the inset of Fig. 2(a): $4 \mathrm{meV}, 5 \mathrm{meV}$, and $<1 \mathrm{meV}$ for ZLG, MLG, and BLG, respectively, at the chemical potential axis), it is important to recall that this does not necessarily imply narrow experimental conditions: a drastic change in the number of $\mathrm{Si}\left(N_{\mathrm{Si}}\right)$ and $\mathrm{C}\left(N_{\mathrm{C}}\right)$ atoms can correspond to a small change of $\mu$. For instance, one would first have to remove all $\mathrm{Si}$ from the $\mathrm{SiC}$ crystal to go beyond the graphite stability line in equilibrium. However, the surface-energy differences between the different phases are also rather small (a few tens of milli electron volts per $(1 \times 1) \mathrm{SiC}$ surface unit cell). The primary approximations that we cannot systematically improve in our calculations are possible small temperature-dependent surface strain effects [49] and the density functional itself. For instance, correlation effects beyond standard semilocal DFT render the Si-rich $(\sqrt{3} \times \sqrt{3})-R 30^{\circ}$ adatom phase nonmetallic [55-57], whereas semilocal DFT gives a metallic interface. Similar Si dangling bonds exist under the ZLG interface. Here, too, the question of a metallic (semilocal DFT) [58] vs (almost) nonmetallic interface in angleresolved photoemission spectroscopy $[13,59]$ has been raised. If so, this nonmetallicity could lead to small shifts in the surface energetics and thus in the phase diagram. Since all phases in question should experience some small additional stabilization, the impact on the relative stability may be even smaller, but current parameter-free density functionals cannot decide to which degree. The key message of Fig. 2(a) is thus that MLG and its related phases all appear at least as very near equilibrium phases, a fact that is nonetheless critical for a qualitatively correct understanding of their growth and properties.

What we can do is to show how our results would be affected by different density functionals. We have thus recomputed the surface phase diagram up to MLG for two widely used functionals in Figs. 2(b) and 2(c): plain PBE and LDA. As expected, the absence of vdW tails in plain PBE changes the phase diagram drastically. Due to the overstabilization of graphite, its stability line moves significantly further to the left, as does the crossover point between ZLG and MLG. Therefore, neither ZLG nor MLG becomes stable over the competing Si-rich $(\sqrt{3} \times \sqrt{3})-R 30^{\circ}$ phase in PBE, in contradiction to experiment [16]. In the LDA, the most significant change compared to $\mathrm{PBE}+\mathrm{vdW}$ is the apparent incorrect stability hierarchy of graphite vs diamond (without ZPC). If the diamond line is discounted, our calculations show the ZLG-MLG crossover point almost exactly on the graphite line. Still, even taking LDA at face value implies the existence of $T-p$ conditions very close to equilibrium for MLG, making the experimental search for such conditions promising.

Figure 2 thus shows the most important point of our Letter: the existence of equilibrium or near-equilibrium chemical potential ranges for ZLG, MLG, and even BLG, corresponding to specific $T / p$ conditions in experiment. This finding proves the potential for much better growth control than what could be expected if each phase were just a necessary (but not thermodynamically stable) kinetic intermediate. While true reversibility for actual MLG may be hard to achieve [16,18] (the reverse growth process, disassembling a fully formed graphene plane, would be kinetically difficult), the forward growth process from MLG to BLG under Si out diffusion should still be limitable by appropriate $T / p$ conditions. A macroscopically homogeneous surface very close to pure-phase MLG should thus be achievable in principle.

Figure 2 shows unambiguously the importance of a consistently accurate numerical treatment of the experimentally observed phases in their large unit cells. It would obviously be much more economical to consider smallercell approximant phases to the true $(6 \sqrt{3} \times 6 \sqrt{3})$ - $R 30^{\circ}$ supercells. However, the residual artificial strain and inadequate bonding in those phases are too large for meaningful surface energy comparisons [60]. For instance, the popular $(\sqrt{3} \times \sqrt{3})-R 30^{\circ}[47,61]$ approximant would intersect the graphite stability line at a surface energy $\left.\gamma\right|_{\mu_{C}=E_{\text {graphite }}^{\text {bul }}}=$ $0.15 \mathrm{eV}$, far above the actually stable phases. Likewise, a slightly rotated $(5 \times 5)$ approximant to ZLG [62] (a periodicity sometimes seen in experiment [17,63]) would intersect at $\left(\left.\gamma\right|_{\mu_{C}=E_{\text {graphite }}^{\text {bulk }}}=-0.35 \mathrm{eV}\right)$, higher by $0.06 \mathrm{eV}$ than even the closest competing Si-rich phase, the $(\sqrt{3} \times \sqrt{3})-R 30^{\circ} \mathrm{Si}$ adatom phase $\left(\left.\gamma\right|_{\mu_{C}=E_{\text {graphite }}^{\text {bulk }}}=\right.$ $-0.41 \mathrm{eV})$. The $(5 \times 5)$ phase is either a nonequilibrium phase, or its structure is not the same as that assumed in Ref. [62].

The true problem with artificially strained approximant phases is that their strain can obscure other electronically relevant properties, such as the energetics of defects. As an example, we consider a $\mathrm{C}$-rich defect type suggested as an equilibrium feature of the ZLG phase in Ref. [58]. Two different defect positions, "hollow" and "top," were proposed [58]. Indeed, both would be more stable than the hypothetical $(\sqrt{3} \times \sqrt{3})-R 30^{\circ}$ ZLG approximant when included there in a $(3 \times 3)$ arrangement as done in Ref. [58]: $-1.75 \mathrm{eV}$ per defect for the hollow position, $-2.93 \mathrm{eV}$ per defect for top, both at $\mu_{C}=E_{\text {graphite }}^{\text {bulk }}$. However, the same defects are unstable when included into and compared to the correct $(6 \sqrt{3} \times 6 \sqrt{3})-R 30^{\circ}$ ZLG phase: $+5.28 \mathrm{eV}$ per defect for the hollow position, $+5.27 \mathrm{eV}$ for the top position, again at $\mu_{C}=E_{\text {graphite }}^{\text {bulk }}$ (see the Supplemental Material [27] for more details).

In conclusion, we can now rationalize some specific growth-related observations. In UHV, the background pressures of $\mathrm{Si}$ and $\mathrm{C}$ are low and ill defined, leading to inferior morphologies and wide variations of experimental conditions [51,64]. Much more homogeneous growth can be achieved in an $\mathrm{Ar}$ atmosphere [3] or by controlling the $\mathrm{Si}$ background pressure by a disilane reservoir [16] or a confined cavity [5]. Maintaining a controlled Si partial 
pressure at constant, higher temperature than allowed in UHV is most likely the important step. Similar considerations may also aid the much more difficult growth of MLG on the $C$ face $[5,65]$, where a well-defined interface layer at finite disilane pressure was proposed [6]. Nearequilibrium surface conditions may indeed be the key to the best possible epitaxial growth of graphene on $\mathrm{SiC}$.

The authors thank Dr. J. Marcelo J. Lopes (Paul-DrudeInstitut für Festkörperelektronik, Berlin) and Dr. Ulrich Starke (Max-Planck-Institut für Festkörperforschung, Stuttgart) for helpful discussions during the preparation of this work. This work was partially supported by the DFG collaborative research project 951 "HIOS".

[1] A. Van Bommel, J. Crombeen, and A. Van Tooren, Surf. Sci. 48, 463 (1975).

[2] I. Forbeaux, J.-M. Themlin, and J.-M. Debever, Phys. Rev. B 58, 16396 (1998).

[3] K. V. Emtsev, A. Bostwick, K. Horn, J. Jobst, G. L. Kellogg, L. Ley, J.L. McChesney, T. Ohta, S. A. Reshanov, J. Röhrl et al., Nat. Mater. 8, 203 (2009).

[4] C. Riedl, C. Coletti, and U. Starke, J. Phys. D 43, 374009 (2010).

[5] W. A. de Heer, C. Berger, M. Ruan, M. Sprinkle, X. Li, Y. $\mathrm{Hu}$, B. Zhang, J. Hankinson, and E. Conrad, Proc. Natl. Acad. Sci. U.S.A. 108, 16900 (2011).

[6] N. Srivastava, G. He, Luxmi, and R. M. Feenstra, Phys. Rev. B 85, 041404 (2012).

[7] G. R. Yazdi, R. Vasiliauskas, T. Iakimov, A. Zakharov, M. Syväjärvi, and R. Yakimova, Carbon 57, 477 (2013).

[8] Y. Lin, A. Valdes-Garcia, S. Han, D. Farmer, I. Meric, Y. Sun, Y. Wu, C. Dimitrakopoulos, A. Grill, P. Avouris et al., Science 332, 1294 (2011).

[9] S. Hertel, D. Waldmann, J. Jobst, A. Albert, M. Albrecht, S. Reshanov, A. Schöner, M. Krieger, and H. Weber, Nat. Commun. 3, 957 (2012)

[10] C. Berger, Z. Song, T. Li, X. Li, A. Ogbazghi, R. Feng, Z. Dai, A. Marchenkov, E. Conrad, N. Phillip et al., J. Phys. Chem. B 108, 19912 (2004).

[11] P. N. First, W. A. de Heer, T. Seyller, C. Berger, J. A. Stroscio, and J.-S. Moon, MRS Bull. 35, 296 (2011).

[12] S.-H. Ji, J. B. Hannon, R. M. Tromp, V. Perebeinos, J. Tersoff, and F. M. Ross, Nat. Mater. 11, 114 (2011).

[13] K. V. Emtsev, F. Speck, T. Seyller, L. Ley, and J. D. Riley, Phys. Rev. B 77, 155303 (2008).

[14] F. Ming and A. Zangwill, Phys. Rev. B 84, 115459 (2011).

[15] K. Reuter and M. Scheffler, Phys. Rev. B 65, 035406 (2001).

[16] R. M. Tromp and J. B. Hannon, Phys. Rev. Lett. 102, 106104 (2009).

[17] C. Riedl, U. Starke, J. Bernhardt, M. Franke, and K. Heinz, Phys. Rev. B 76, 245406 (2007).

[18] J. B. Hannon (private communication).

[19] A. Tkatchenko and M. Scheffler, Phys. Rev. Lett. 102, 073005 (2009).

[20] J. P. Perdew, K. Burke, and M. Ernzerhof, Phys. Rev. Lett. 78, 1396 (1997)
[21] S. Grimme, Comput. Mol. Sci. 1, 211 (2011).

[22] M. Dion, H. Rydberg, E. Schröder, D. C. Langreth, and B. I. Lundqvist, Phys. Rev. Lett. 92, 246401 (2004).

[23] K. Lee, É. D. Murray, L. Kong, B. I. Lundqvist, and D. C. Langreth, Phys. Rev. B 82, 081101 (2010).

[24] J. Klimes, D. R. Bowler, and A. Michaelides, J. Phys. Condens. Matter 22, 022201 (2010).

[25] G. X. Zhang, A. Tkatchenko, J. Paier, H. Appel, and M. Scheffler, Phys. Rev. Lett. 107, 245501 (2011).

[26] A. Tkatchenko, R. A. DiStasio, R. Car, and M. Scheffler, Phys. Rev. Lett. 108, 236402 (2012).

[27] See Supplemental Material at http://link.aps.org/ supplemental/10.1103/PhysRevLett.111.065502. The Supplemental Material includes reference data for the numerical convergence of our calculations, calculated lattice parameters, and other cohesive properties of the bulk reference phases $3 \mathrm{C}$-SiC, diamond $\mathrm{Si}$, diamond and graphite $\mathrm{C}$, without and with zero-point corrections for the functionals used here, and structural details of the defects as introduced in Ref. [58].

[28] Z. Li and R. C. Bradt, J. Mater. Sci. 21, 4366 (1986).

[29] V. Blum, R. Gehrke, F. Hanke, P. Havu, V. Havu, X. Ren, K. Reuter, and M. Scheffler, Comput. Phys. Commun. 180, 2175 (2009).

[30] V. Havu, V. Blum, P. Havu, and M. Scheffler, J. Comput. Phys. 228, 8367 (2009).

[31] T. Auckenthaler, V. Blum, H. Bungartz, T. Huckle, R. Johanni, L. Krämer, B. Lang, H. Lederer, and P. Willems, Parallel Comput. 37, 783 (2011).

[32] U. Starke, J. Schardt, J. Bernhardt, M. Franke, K. Reuter, H. Wedler, K. Heinz, J. Furthmüller, P. Käckell, and F. Bechstedt, Phys. Rev. Lett. 80, 758 (1998).

[33] R. Kaplan, Surf. Sci. 215, 111 (1989).

[34] U. Starke, J. Schardt, J. Bernhardt, M. Franke, and K. Heinz, Phys. Rev. Lett. 82, 2107 (1999).

[35] Y. Baskin and L. Meyer, Phys. Rev. 100, 544 (1955).

[36] T. Seyller, A. Bostwick, K. Emtsev, K. Horn, L. Ley, J. McChesney, T. Ohta, J. Riley, E. Rotenberg, and F. Speck, Phys. Status Solidi B 245, 1436 (2008).

[37] J. B. Hannon, M. Copel, and R. M. Tromp, Phys. Rev. Lett. 107, 166101 (2011).

[38] T. Ohta, A. Bostwick, T. Seyller, K. Horn, and E. Rotenberg, Science 313, 951 (2006).

[39] S. Kim, J. Ihm, H. J. Choi, and Y. W. Son, Phys. Rev. Lett. 100, 176802 (2008).

[40] F. Varchon, P. Mallet, J.-Y. Veuillen, and L. Magaud, Phys. Rev. B 77, 235412 (2008).

[41] G. Sclauzero and A. Pasquarello, Phys. Rev. B 85, 161405 (R) (2012)

[42] W. Chen, H. Xu, L. Liu, X. Gao, D. Qi, G. Peng, S. C. Tan, Y. Feng, K. P. Loh, and A. T. S. Wee, Surf. Sci. 596, 176 (2005).

[43] C. Berger, J. Veuillen, L. Magaud, P. Mallet, V. Olevano, M. Orlita, P. Plochocka, C. Faugeras, G. Martinez, M. Potemski et al., Int. J. Nanotechnology 7, 383 (2010).

[44] S. Goler, C. Coletti, V. Piazza, P. Pingue, F. Colangelo, V. Pellegrini, K. V. Emtsev, S. Forti, U. Starke, F. Beltram et al., Carbon 51, 249 (2013).

[45] L. H. de Lima, A. de Siervo, R. Landers, G. A. Viana, A. M. B. Goncalves, R. G. Lacerda, and P. Häberle, Phys. Rev. B 87, 081403(R) (2013). 
[46] W. Norimatsu and M. Kusunoki, Chem. Phys. Lett. 468, 52 (2009).

[47] F. Varchon, R. Feng, J. Hass, X. Li, B. Nguyen, C. Naud, P. Mallet, J. Veuillen, C. Berger, E. Conrad et al., Phys. Rev. Lett. 99, 126805 (2007).

[48] From a microscopic point of view, as long as the Si diffusion path to the outside (sublimation) remains kinetically accessible, so does the inward diffusion path, and near equilibrium with a $\mathrm{Si}$ reservoir gas can be achieved. During intermediate stages of the formation of a new graphene plane [46], such diffusion paths must be available.

[49] J. Roehrl, M. Hundhausen, K. Emtsev, T. Seyller, R. Graupner, and L. Ley, Appl. Phys. Lett. 92, 201918 (2008).

[50] In principle, we could include the much smaller $(p, T)$ dependence of the solid phases by focusing on Gibbs free energies $G(T, p)$ in the (quasi)harmonic approximation instead of $E$. However, quantifying this $T$ dependence precisely would here necessitate accurate phonon calculations for structure sizes of the order of $\approx 2000$ atoms, a task that is computationally prohibitive at present. ZPC are small for the bulk phases (see the Supplemental Material [27]).

[51] W. Lu, J. J. Boeckl, and W. C. Mitchell, J. Phys. D 43, 374004 (2010).

[52] R. Berman and F. Simon, Z. Elektrochem.: Ber. Bunsenges. Phys. Chem. 59, 333 (1955).
[53] M. T. Yin and M. L. Cohen, Phys. Rev. B 29, 6996 (1984).

[54] F. Lazarevic, L. Nemec, V. Blum, and M. Scheffler (to be published).

[55] J.E. Northrup and J. Neugebauer, Phys. Rev. B 52, R17001 (1995).

[56] M. Rohlfing and J. Pollmann, Phys. Rev. Lett. 84, 135 (2000).

[57] F. Bechstedt and J. Furthmüller, J. Phys. Condens. Matter 16, S1 721 (2004).

[58] Y. Qi, S. H. Rhim, G. F. Sun, M. Weinert, and L. Li, Phys. Rev. Lett. 105, 085502 (2010).

[59] K. Emtsev, T. Seyller, F. Speck, L. Ley, P. Stojanov, J. Riley, and R. Leckey, Mater. Sci. Forum 556-557, 525 (2007).

[60] G. Sclauzero and A. Pasquarello, Diam. Relat. Mater. 23, 178 (2012).

[61] A. Mattausch and O. Pankratov, Phys. Rev. Lett. 99, 076802 (2007).

[62] O. Pankratov, S. Hensel, and M. Bockstedte, Phys. Rev. B 82, 121416 (2010).

[63] U. Starke and C. Riedl, J. Phys. Condens. Matter 21, 134016 (2009).

[64] P. Sutter, Nat. Mater. 8, 171 (2009).

[65] J. Hass, R. Feng, J. E. Millán-Otoya, X. Li, M. Sprinkle, P. N. First, W. A. de Heer, E. H. Conrad, and C. Berger, Phys. Rev. B 75, 214109 (2007). 